\title{
THE PEAT DEPOSITS OF NEW JERSEY.
}

\section{HENRY B. KüMMEL.}

Recent investigations ${ }^{1}$ by members of the Geological Survey of New Jersey have made available much detailed information regarding both the occurrence and the quality of the peat deposits of northern New Jersey.

Occurrence.-As is shown by the field work of Mr. McCourt

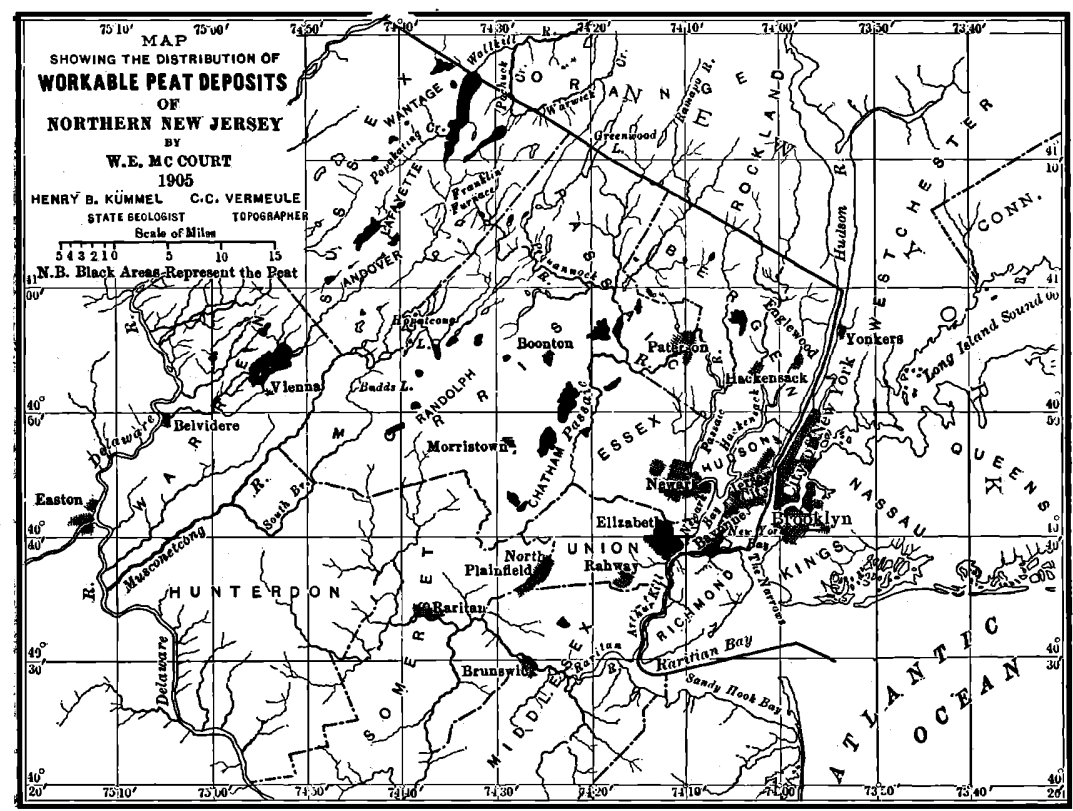

Fig. I. Sketch map of Northern New Jersey showing distribution of workable peat deposits. (After McCourt.)

(see Fig. I), peat beds of good quality and of considerable extent occur in Bergen, Morris, Sussex and Warren counties. Not all the swamps of these counties are peat swamps, but extensive de-

" A Report on the Peat Deposits of Northern New Jersey," by C. 'W. Parmelee and W. E. McCourt. Annual Report of the State Geologist for I905. 
posits were found near Allendale and along Sprout Brook, in Bergen County; in the Black Meadows, Troy Meadows, and the Bog and Vly Meadows in Morris; the Vernon and Sussex meadows in Sussex; and the Pequest Meadows in Warren County. These deposits each range from I 50 to 2,000 or 3,000 acres in surface area and depths of 8 to Io feet were not infrequently found, although probably the average depth is less than this. Two somewhat distinct types of peat occur, a brown fibrous variety and a black, waxy, spongy one, but these varieties are not sharply differentiated from each other. The latter is the older and where both occur in one bog is found under the more fibrous form.

Moisture.-As is well known, all peat contains a large amount of water when first dug, which in some cases amounts to as much as 85 or 90 per cent. When exposed to the air, a large portion of the water evaporates, but there always remains some which cannot be driven out except at higher temperatures. It is manifest that the value of peat as fuel depends considerably upon the extent to which this moisture can be expelled by economical means for not only does each unit of the water replace a unit of combustible material, but a certain amount of the heat generated is consumed in vaporizing the moisture remaining. Consequently peat containing 25 per cent. of moisture, which should be the maximum for a good air-dried peat to be used as fuel, has something less than 75 per cent. of the heating power of a perfectly dry peat.

In this investigation the moisture content of I07 samples of air-dried peat was determined by Dr. Parmelee. The samples were spread out in thin layers, and exposed for several days to the room temperature. "A small portion, representing an average sample, was carefully taken and ground through a mill to such a fineness that it would all pass through a Ioo-mesh sieve. Two watch glasses ground on the edges to fit tightly together, with a brass spring to hold them firmly, were accurately weighed, and then five grams of the powdered sample were weighed and placed in one glass. This was placed in an oven which was kept at a temperature of $105^{\circ} \mathrm{C}$. and the sample left there until, 
by repeated drying and weighing, no further loss of moisture was found. This operation generally required 96 hours. The weighings were always undertaken with the cover glass securely clamped over the sample by means of the spring. This was found necessary because of the very hygroscopic nature of the dry powder. The samples showed a wide range. The lowest amount of moisture in an individual sample was 6.09 per cent. The highest was 28.I4 per cent. Considering the whole number of samples, it may be said that as the ash content increases the moisture content is lower; although the converse is not true, that a high moisture content indicates a low ash. It is not safe to apply the general rule to particular cases. The average moisture content of IO 7 samples having 50 per cent. or less of ash was found to be $15.5^{2}$ per cent." 1

Ash.-The amount of ash in peat is also of great importance in determining its value as a fuel. Not only is there the loss of the combustible material which is displaced by the ash, but of a certain amount of heat which is used in bringing the noncombustible matter to the temperature of the fire and maintaining the heat at that point. Furthermore, the cost of digging and transporting this ash has to be paid. As shown by the laboratory experiments, the ash in the samples tested varies greatly in amount, and is by no means always the same in peats which outwardly resemble each other closely.

The amount of ash was determined from the oven-dried sample, which was kept in a tightly-corked test-tube. A small amount, approximately I gram, was poured into a weighed platinum crucible,- - the exact amount being determined by the difference in weight of the test-tube before and after. Ignition was carried out over a gas flame in the usual way and the residue weighed as ash. The percentage of ash was calculated on the basis of the air-dried material in order to obtain results comparable to what would occur under economic conditions. One hundred and seven samples were thus tested and the amount of ash was found to range from 5.04 to over 50 per cent.; those containing more than the latter figure not deserving the name of peat.

$$
{ }^{1} \text { Loc. cit., p. } 257 .
$$


Calorimeter Tests.—" The calorimetric tests of the New Jersey peats were obtained by the use of the Standard or Parr calorimeter. This apparatus consists of a hollow cylinder or bomb which may be tightly closed at either end. For a test, the bomb is charged with a small quantity of the powdered dry sample, crushed to pass through a Ioo mesh. The quantity varied from one half to three fourths of a gram. This was mixed with sodium peroxide and potassium chlorate which furnished oxygen for combustion. The bomb, after filling, was immersed in a large cylinder containing a quantity of water-two liters. The large cylinder was placed in a fiber bucket having a wall between the large cylinder and the outer wall of the bucket. This arrangement provided an insulating air space between the large cylinder and the wall, and another between the wall and the bucket, which provision prevented temperature changes due to external causes. The bomb, when immersed, stood vertical, centered upon a pin so as to rotate easily. Four vanes attached to the sides, and an axle or stem of small size projected from the top of the bomb upwards. On the upper part of the stem a small pulley was attached, which was driven by a belt from a smaller motor. Within the bomb there were two metal poles attached to the cover and connected by a loop of fine iron wire of sufficient length to dip into the charge. This loop was heated to incandescence by the passage of an electric current, which thus ignited the charge. The completeness of - the combustion is assured by the presence of the oxidizing chemicals mixed with the sample. The heat generated by this reaction radiates from the bomb and heats the surrounding water. By means of a delicate thermometer the rise in the temperature of the water could be noted and the calorific value calculated.

Since the determinations were made on samples dried at a temperature of $105^{\circ} \mathrm{C}$., the results are higher than may be expected from the air-dried peat. The advantage of this method however is that all the samples may be more accurately compared since the amount of moisture remaining after air drying is dependent upon local conditions. Only those samples having 25 per cent or less of ash were tested. All tests were run in 
duplicate and the results in all but two instances differed by less than one division on the thermometer used. That is, less than one twentieth of $I^{\circ} \mathrm{F}$. In many instances the calculated difference was less than one two-hundredths of $\mathrm{I}^{\circ} \mathrm{F}$.

The results obtained by the use of this apparatus have not been checked by comparison with the results on the same sample using other types of the calorimeter. Since however it is known that this instrument gives accurate results with very similar fuels, such as lignite, brown coal, napthalene, etc., it is quite unlikely that the error from this source is greater than the experimental."1

Sixty-one samples were tested in duplicate, and results ranging from 5,876 calories as a maximum to 4,026 calories as a minimum were obtained. From a comparison of the detailed figures given in the table below it will be seen that with but few exceptions high calories accompany low ash, as was to be expected. The explanation of the exceptions does not appear from the facts presented but might be revealed by an ultimate chemical analysis.

In connection with these tests it is of interest to note the calorimetric value of some coals recently tested :

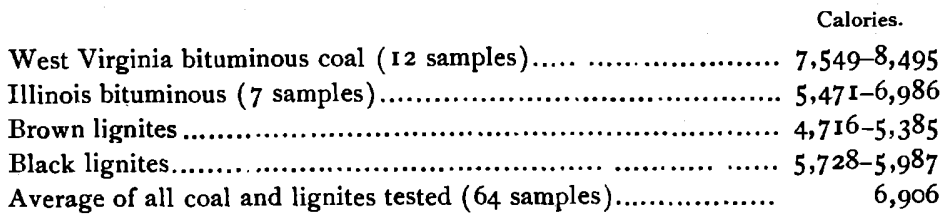

In making this comparison, however, it must be born in mind that the coal tests were made on air-dried samples, whereas the peat tests were made on samples dried at $105^{\circ} \mathrm{C}$. This difference in procedure discriminates against the coal to a certain extent, so that the peat makes a better showing relatively than would have been the case had both sets of tests been made on the same basis. The greater the percentage of water present in the peat samples, the greater does this discrimination become,

${ }^{1}$ Loc. cit., p. 259.

${ }^{2}$ Parker, Holmes, Campbell, et al. U. S. G. S. Professional Paper 48, p. I 96 et seq. 
and it is necessary, therefore, in using the figures given in the table below to make allowance for the varying percentages of moisture present. In cases where the moisture is as much as 25 per cent., the calories should be diminished by nearly 30 per cent., to obtain results comparable with the coal test cited above.

Coking Tests.-Tests were made by Mr. R. B. Gage, chemist of the survey, for the purpose of securing data to indicate which of the New Jersey peats might be used in the manufacture of peat coke, and to determine the amount of volatile gases and fixed carbon in the peat. The standard method of determination was employed, $i$. e., to heat I gram of the air-dried powdered peat in a platinum crucible for $3^{\mathrm{T} / 2}$ minutes over a bunsen burner with a low flame, which was then turned on full for the same length of time after which the crucible was cooled and weighed. The results are tabulated below. Since by this method the ash was included in the coke and weighed as such, samples containing high ash gave correspondingly high results for coke. It is necessary, therefore, to consider the ash content in comparing the figures for the coke.

Nitrogen Content.- " All the samples were examined by the Kjehldahl method for the total nitrogen present. Three grams of the powdered air-dried sample were found sufficient. These results may be useful for the manufacturer of filler for fertilizer, and the gas manufacturer using peat either in a by-product coking oven or with the Mond producer or similar systems for the recovery of the nitrogen. In our examination of I23 samples from this state, the lowest amount of nitrogen found was 0.74 per cent., the highest figure was 2.83 per cent. The average was 1.75 per cent. The results of the analyses are given in the table below."'

${ }^{1}$ Parmelee and McCourt, loc. cit., p. 262. 
TABLE SHOWING ALL TESTS OF NEW JERSEY PEATS.

\begin{tabular}{|c|c|c|c|c|c|c|c|c|c|c|}
\hline Locality. & 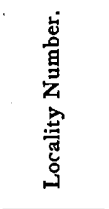 & 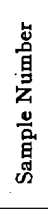 & 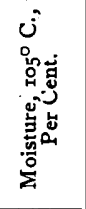 & 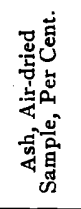 & 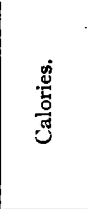 & 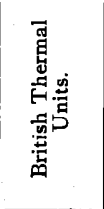 & 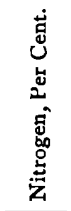 & 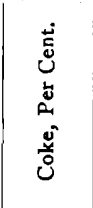 & 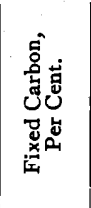 & 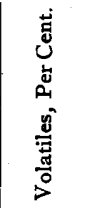 \\
\hline equannoc & & I & 09 & 10.91 & 4,966 & 8, & 2. 18 & 38.23 & 20 & 61.77 \\
\hline dale.. & $5^{a}$ & 2 & 15.00 & I 8.89 & & & 2.I 3 & 41.57 & 22.68 & 58.43 \\
\hline endale... & $5 \mathrm{ab}$ & 3 & 14.11 & 5.04 & 5,896 & $10,6 \mathrm{I} I$ & 2.17 & 29.20 & 24.16 & 70.80 \\
\hline prout Broo & 8 a \& b & 4 & 16.42 & 64.17 & ........... & & 1.60 & ( & ......... & \\
\hline prout Broo & $8 \mathrm{~b}$ & 5 & 30.70 & 29.12 & , n.......... & & 1.90 & & & \\
\hline prout $\mathrm{Broo}$ & $8 \mathrm{c}$ & 7 & 9.08 & 50.43 & & & 0.95 & & & \\
\hline ckensack & $9 \mathrm{~b}$ & 8 & 4.93 & 72.49 & .............. & $\cdots$ & כליטג & & & \\
\hline ensach & $9 \mathrm{c}$ & 9 & 14.20 & 20.74 & $4,3^{12}$ & $7,76 \mathrm{I}$ & I. 87 & 46.75 & 26.01 & 53.25 \\
\hline dale & IO b & IO & 7.25 & $57 \cdot 55$ & $2,23^{8}$ & 4,028 & I. 3 I & ........ & .......... & \\
\hline dale. & $10 \mathrm{e}$ & I I & 13.28 & 24.43 & 4,041 & 7,274 & 2.40 & ............. & …......... & \\
\hline aire $F$ & I I a & 12 & 6.31 & 67.70 & .......... & & 0.82 & & & \\
\hline$\ldots$ & $12 \mathrm{a}$ & 13 & 7.20 & 64.24 & & ..... & I.05 & .......... & .......... & \\
\hline & I 2 a & 14 & I 1.89 & 30.54 & .......... & & 1.70 & $\ldots$. & & \\
\hline $\mathrm{Ne}$ & $12 \mathrm{~d}$ & 15 & 14.16 & 10. 34 & 5,187 & $9,33^{8}$ & 2.24 & 33.09 & 22.75 & 66.91 \\
\hline $\mathrm{Ner}$ & $12 \mathrm{e}$ & I6 & 12.84 & 23.78 & 4,212 & 7,582 & 2.06 & 43.73 & I9.95 & 56.27 \\
\hline$\ldots$. & I $3 \mathrm{ef}$ & I 7 & 8.77 & 53.87 & 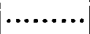 & ......... & I. 52 & .......... & & \\
\hline le .. & $14 \mathrm{e}$ & 18 & $7 \cdot 49$ & 61.80 & & & 0.88 & & & \\
\hline te . & $15 \mathrm{e}$ & I9 & 12.26 & 23.29 & 4,092 & 7,365 & 2.01 & 44.85 & 21.56 & 55.15 \\
\hline te & $15 \mathrm{e}$ & 2 I & 9.64 & 26.12 & & & I. $5^{8}$ & & & \\
\hline tte & I $5 \mathrm{~b}$ & 22 & 18.27 & 10.45 & 5,004 & $9, \infty 07$ & 2.83 & $34.9 \mathrm{I}$ & 24.46 & 65.09 \\
\hline Jun & I6 a & 23 & 27.56 & 20.88 & 4,026 & 7,247 & I. 99 & 40.20 & 19.32 & 59.80 \\
\hline Jun & $16 a$ & 24 & 22.34 & 32.76 & $\cdots$ & $\cdots$ & 1.65 & $\ldots$ & ........... & $\cdots \cdots$ \\
\hline rta Jun & $16 \mathrm{~d}$ & 25 & 9.73 & 29.22 & & & 2.37 & & $-\mathrm{s}+2$ & \\
\hline Jun & $16 \mathrm{e}$ & 26 & 6 & 46.83 & & & I. 53 & & & \\
\hline ngto & 17 a & 27 & I6.06 & 37.26 & & $\ldots$ & 1.42 & & ...... & \\
\hline hingtor & I 7 e & 28 & 14.28 & $33 \cdot 7^{2}$ & & & I. 20 & & & \\
\hline ............... & $20 \mathrm{e}$ & 29 & 13.84 & 26.23 & .. & & 2.34 & ...... & & \\
\hline Sussex & $20 \mathrm{f}$ & 30 & 9.73 & 46.24 & & & I. 78 & & & \\
\hline Sussex & $20 \mathrm{~g}$ & $3^{I}$ & $24 \cdot 30$ & 20. II & 4,165 & 7,496 & I. 78 & 41.90 & 21.79 & 58.10 \\
\hline $\operatorname{sex} \ldots$ & $20 \mathrm{~h}$ & 32 & I 2.89 & 51.06 & .... & ... & I. $3^{\circ}$ & $\cdots \cdot$ & & \\
\hline Sussex .... & 201 & 33 & IO. 14 & 49.09 & & & I. 59 & & & \\
\hline$e x$ & $20 \mathrm{~m}$ & 34 & I 5.99 & I I. $3^{8}$ & 4,946 & 8,902 & 2.34 & 37.95 & 26.57 & 62.05 \\
\hline ervill & 21 a & 35 & I4. 12 & 17.23 & 4,814 & 8,667 & I. 53 & 41.40 & 24.17 & 58.60 \\
\hline ville & $21 \mathrm{e}$ & 36 & 14.20 & 20.82 & 4,199 & $7,55^{8}$ & I. 86 & $39 \cdot 3^{8}$ & 18.56 & 60.62 \\
\hline ville & $21 \mathrm{e}$ & 40 & I $2.5^{\circ}$ & 23.03 & 4,2 I 2 & 7,582 & I. 77 & $44 \cdot 35$ & 21.32 & 55.65 \\
\hline ville & $21 \mathrm{~g}$ & 4I & I0.69 & 38.08 & & & I. 65 & & & \\
\hline town & $21 \mathrm{i}$ & 42 & I 4.55 & 22.81 & 4,377 & 7,879 & $1.7 \mathrm{I}$ & 44. I 8 & 21.37 & 55.82 \\
\hline 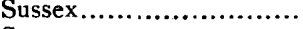 & 200 & 43 & I 4.45 & 28.69 & & & 2. 10 & & & \\
\hline 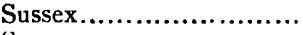 & 200 & 44 & $7 \cdot 57$ & 7.27 & $4,5^{89}$ & 8,260 & 2.00 & 36. I I & 28.84 & 63.89 \\
\hline 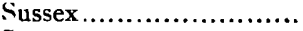 & $20 \mathrm{st}$ & 45 & 9.50 & 56.35 & & & I. 48 & ........... & & ......... \\
\hline & $20 \mathrm{t}$ & 46 & I6.66 & 14.59 & & 8,779 & 2. I3 & 39.77 & 25.18 & 60.23 \\
\hline kle & $22 \mathrm{a}$ & 47 & I4.0I & 13.04 & 4,986 & 8,975 & 2.25 & 40.01 & 26.97 & 59.99 \\
\hline $\mathrm{rt}$ & $22 \mathrm{~b}$ & 48 & $17 \cdot 52$ & I I.36 & 4,908 & 8,834 & 2.02 & 37.53 & 26.17 & 62.47 \\
\hline & $23 a$ & 49 & 12.98 & 38.69 & & .......... & I. 7 I & & ・. & \\
\hline & $23 \mathrm{e}$ & 50 & 12. 55 & 46.27 & & & 1.76 & & & \\
\hline V & $23 \mathrm{~h}$ & $5^{1}$ & II.09 & 50.15 & & & 1.04 & & & \\
\hline$\cdots$ & $23 \mathrm{j}$ & 52 & 13.74 & 36.91 & & & I. 79 & & & \\
\hline 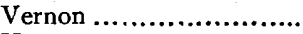 & $23 \mathrm{r}$ & 53 & & 25.26 & & & 2.46 & & & \\
\hline Vernon ....................... & $23 \mathrm{q}$ & 54 & 18.55 & 14.40 & 4,707 & 8,473 & 2.73 & 36.92 & 22.52 & 63.08 \\
\hline
\end{tabular}


TABLE SHOWING ALL TESTS OF NEW JERSEY PEATS.-Continued.

\begin{tabular}{|c|c|c|c|c|c|c|c|c|c|c|}
\hline Locality. & 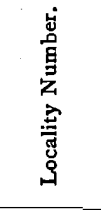 & 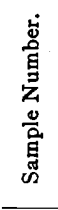 & 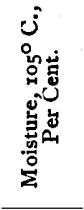 & 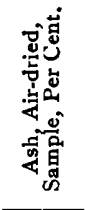 & ט & 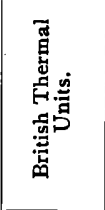 & 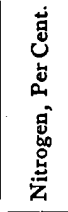 & 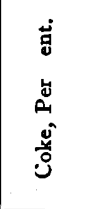 & 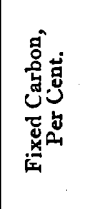 & 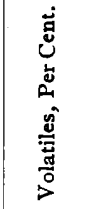 \\
\hline Beaver Run & $25 \mathrm{~d}$ & 55 & 15.70 & 25.86 & & & 2.03 & & & \\
\hline Beaver Run & $25 \mathrm{e}$ & 56 & 14.42 & 28.08 & & & $\begin{array}{l}2.05 \\
1.90\end{array}$ & & & \\
\hline Harmonyval & $25 \mathrm{~g}$ & 57 & 16.66 & 23.25 & 4,027 & 7,249 & 2.20 & 42.60 & 19.35 & 57.40 \\
\hline Stockholm & $26 \mathrm{a}$ & 58 & 17.49 & $5 \cdot 75$ & 5,284 & 9,5 IO & 2.10 & $35 \cdot 3 \mathrm{I}$ & 29.56 & 64.69 \\
\hline Dunker Por & $26 \mathrm{~d}$ & 59 & 17.80 & 6.24 & 5,265 & 9,482 & 2. II & $34 \cdot 30$ & 28.06 & 65.70 \\
\hline off .. & $27 \mathrm{~b}$ & 60 & I6. 77 & 24.70 & 4,049 & 7,287 & 1.98 & 45.90 & 21.20 & 54. 10 \\
\hline Berkshire I & $27 \mathrm{~h}$ & $6 I$ & 15.43 & 18.21 & $4,3^{87}$ & 7,897 & I. 80 & 46.26 & 28.05 & 53.74 \\
\hline aire & $28 a$ & 62 & I 1.87 & $37 \cdot 42$ & a.......... & & I. 78 & & & \\
\hline iire & $28 \mathrm{~b}$ & 63 & I3.87 & 24.95 & 4,276 & 7,697 & I. 79 & 47.10 & 22.15 & 52.90 \\
\hline$\cdots$ & $28 \mathrm{c}$ & 64 & 14.69 & 23.24 & 4,456 & 8,021 & 1.61 & 46.01 & 22.77 & 53.99 \\
\hline Ber & $28 c$ & 65 & 14.04 & 20. Io & 4,800 & 8,640 & I. 54 & 41.95 & 21.85 & 58.05 \\
\hline Berk & $28 \mathrm{bl}$ & 66 & 13.63 & $24 \cdot 3^{8}$ & 4,300 & 7,739 & I. 48 & 45.20 & 20.82 & 54.80 \\
\hline $\begin{array}{l}\text { Mt. } \\
\text { Mt. }\end{array}$ & $29 \mathrm{c}$ & 67 & 14.32 & I6.44 & 5,117 & 9,2 I I & I. 54 & 42.65 & 26.21 & $57 \cdot 35$ \\
\hline $\begin{array}{l}\text { Mt. Ho } \\
\text { Mt. HoI }\end{array}$ & $29 \mathrm{~d}$ & 68 & 15.26 & 5.14 & 5,645 & 10,162 & 1.27 & 43.85 & $3^{88.7} \mathbf{I}$ & 56.15 \\
\hline $\begin{array}{l}\text { Mt. Hope . } \\
\text { Mt. Hope . }\end{array}$ & $29 \mathrm{k}$ & 69 & I 3.60 & 27.46 & & & 1.60 & 2876 & & 6 \\
\hline Kenvil ..... & $\begin{array}{l}29 \mathrm{w} \\
3 \mathrm{I} \mathrm{h}\end{array}$ & $\begin{array}{l}70 \\
71\end{array}$ & $\begin{array}{l}10.27 \\
15.79\end{array}$ & $\begin{array}{r}9.47 \\
19.27\end{array}$ & $\begin{array}{l}5,159 \\
4,355\end{array}$ & $\begin{array}{l}9,287 \\
7,803\end{array}$ & I.87 & $3^{8.76}$ & 29.29 & 61.24 \\
\hline Irc & $32 \mathrm{i}$ & 72 & 14.59 & 9.34 & $\begin{array}{l}\text { 4,J33 } \\
5,488\end{array}$ & $\begin{array}{l}6,003 \\
9,877\end{array}$ & $\begin{array}{l}2.34 \\
\text { I. I } 7\end{array}$ & $\begin{array}{l}42.94 \\
37.64\end{array}$ & $\begin{array}{l}23.67 \\
28.30\end{array}$ & 57.06 \\
\hline Irc & $3^{2 j}$ & 73 & 13.69 & 14.26 & 5,193 & 9,347 & 1.30 & $\begin{array}{l}37.04 \\
41.78\end{array}$ & \begin{tabular}{|l|}
20.30 \\
27.52
\end{tabular} & $62.3^{6}$ \\
\hline & $3^{2} q$ & 74 & 11.80 & 31.40 & …‥, & .......... & I. 42 & & $27.5^{2}$ & 58.22 \\
\hline vay & $33 \mathrm{~g}$ & 75 & I6.20 & 10.54 & 5,057 & 9,102 & 2.1 I & 32.25 & 21.71 & 67.75 \\
\hline vay & $33 \mathrm{~g}$ & 76 & 14.17 & 16.46 & 5,049 & 9,088 & I. 88 & 41.10 & 24.64 & $\begin{array}{l}67.75 \\
58.00\end{array}$ \\
\hline in Vi & 46 & 77 & 10.44 & 51.65 & ............ & $\ldots \ldots \ldots \ldots$ & I. 22 & & & 58.90 \\
\hline m.. & 351 & 80 & 15.21 & 33.07 & .......... & .............. & 1.50 & & & n........... \\
\hline ... & $35 \mathrm{lk}$ & $8 I$ & 14.82 & 53.87 & .......... & $\ldots$ & 0.98 & & & \\
\hline ... & $36 \mathrm{i}$ & 82 & I 2.88 & 34.68 & ......... & ... & I. 59 & & & \\
\hline Mea & $37 \mathrm{~d}$ & 83 & 17.80 & 12.43 & $4,79 \mathrm{I}$ & 8,624 & 2.05 & $3^{8.8} \mathrm{I}$ & $26.3^{8}$ & 61.19 \\
\hline Mead & $37 \mathrm{e}$ & 84 & $17.8 \mathrm{I}$ & 10.84 & 4,885 & 8,794 & I. 88 & 37.45 & $26.6 \mathrm{I}$ & 62.55 \\
\hline [eadows ... & $4 \mathrm{Ib}$ & 85 & I I. 42 & 49. I8 & ... & ... & I. 25 & $\cdots$ & ......... & 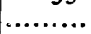 \\
\hline .... & $3^{8 b}$ & 86 & $7 \cdot 5^{8}$ & 66.40 & .. & .... & 0.74 & .......... & & an... \\
\hline$\ldots$ & $38 \mathrm{f}$ & 87 & 9.59 & $50.4^{8}$ & …........ & & 1.26 & & & ....... \\
\hline Gr & $38 \mathrm{i}$ & 88 & II. 80 & 45.92 & $\ldots \ldots \ldots$ & & I. 34 & & & ............. \\
\hline t Swa & $3^{81}$ & 89 & I6.19 & 13.86 & 4,947 & 8,905 & 2.07 & 40.09 & 26.23 & 59.91 \\
\hline Troy Meado & 4 I i & 90 & I 3.27 & 39.02 & ......... & ..... & I. 36 & $\cdots$ & c......... & …...... \\
\hline Troy Mead & 411 & 9 I & I 3.94 & $32.6 \mathrm{I}$ & ... & ........... & I. 65 & & & $\ldots \ldots$ \\
\hline Troy Meado & $41 s$ & 92 & I 7.54 & 10.60 & 5,272 & 9,490 & I. 46 & 37.80 & 27.20 & 62.20 \\
\hline Troy Meado & 4 I b & 93 & 17.86 & I 3.44 & 4,901 & 8,820 & I. 98 & $3^{8.96}$ & $25 \cdot 52$ & 61.04 \\
\hline ...... & 42 a & 94 & 17.89 & I 7.6 I & 4,575 & 8,237 & I.80 & 42.29 & 24.68 & $57.7 \mathrm{I}$ \\
\hline p............. & $42 q$ & 95 & I3. I 5 & 39.89 & $\cdots \cdots$ & ........... & I. 40 & ... & & ......... \\
\hline$\cdots$ & $43^{a}$ & 97 & I 4.94 & $29.4 \mathrm{I}$ & & & I. 74 & 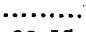 & & \\
\hline noc & $45 \mathrm{c}$ & 98 & I 9.84 & I 2.69 & 4,789 & 8,620 & I.6I & 39.15 & 26.46 & 60.85 \\
\hline & & 100 & II.68 & 36.87 & $\cdots$ & & I.33 & $\cdots$ & & $\cdots \cdots \cdots$ \\
\hline a $V$ & $47 \mathrm{e}$ & $\begin{array}{l}101 \\
\text { IOI }\end{array}$ & I5.26 & $\begin{array}{r}25.19 \\
708\end{array}$ & 5278 & & I. 58 & & & \\
\hline & $\begin{array}{l}47 \mathrm{~g} \\
48 \mathrm{c}\end{array}$ & 102 & $\begin{array}{l}17.25 \\
14.18\end{array}$ & $\begin{array}{r}7.20 \\
22.27\end{array}$ & 5,370 & & 2.16 & 37.08 & 29.80 & 62.92 \\
\hline y $\mathrm{Ri}$ & $48 \mathrm{f}$ & 104 & $\begin{array}{l}14.10 \\
14.73\end{array}$ & 26.66 & $\ldots$ & $\ldots$ & $\begin{array}{l}1.50 \\
1.76\end{array}$ & & & \\
\hline Dismal Swamp.... & 50 & 105 & 19.02 & 20.43 & 4,335 & $7,80_{3}$ & 1.70 & 42.35 & 21.92 & 57.65 \\
\hline Mea & & I06 & 21.33 & 50.86 & & & 0.86 & & & ........ \\
\hline low ... & $52 \mathrm{D}$ & 107 & 11.00 & 44.34 & $\cdots \ldots \ldots$ & & I. 24 & & & \\
\hline
\end{tabular}


TABLE SHOWING ALL TESTS OF NEW JERSEY PEATS.—Concluded.

\begin{tabular}{|c|c|c|c|c|c|c|c|c|c|c|}
\hline Locality. & 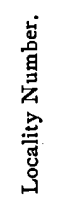 & 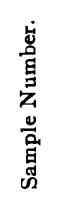 & 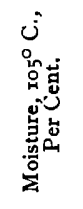 & 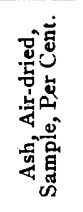 & 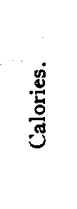 & 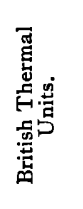 & 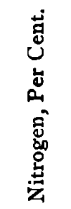 & 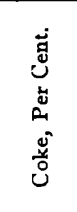 & 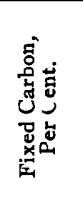 & 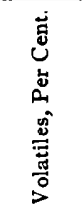 \\
\hline Newark Meadow & $53 \mathrm{k}$ & 108 & I8.59 & 20.06 & 4,347 & 7,833 & \multirow{6}{*}{$\begin{array}{l}1.92 \\
0.78 \\
1.98 \\
1.96 \\
1.62 \\
1.28 \\
1.82\end{array}$} & $43 \cdot 55$ & 23.49 & 56.45 \\
\hline Elizabethport . & $54 \mathrm{e}$ & 109 & 9.02 & 64.66 & & & & & & \\
\hline $\begin{array}{l}\text { Danville ........... } \\
\text { Danville ......... }\end{array}$ & $\begin{array}{l}56 \mathrm{f} \\
56 \mathrm{z}\end{array}$ & $\begin{array}{l}\text { IIO } \\
\text { I I I }\end{array}$ & $\begin{array}{l}20.84 \\
27.70\end{array}$ & $\begin{array}{l}\text { I I,04 } \\
\text { I3.3I }\end{array}$ & $\begin{array}{l}5,234 \\
4,562\end{array}$ & $\begin{array}{l}9,42 \mathrm{I} \\
8,213\end{array}$ & & $\begin{array}{l}36.90 \\
40.26\end{array}$ & $\begin{array}{l}25.80 \\
26.95\end{array}$ & $\begin{array}{l}03.10 \\
59.74\end{array}$ \\
\hline Southtowr & $58 a$ & II 2 & 24.07 & 6.54 & 5,098 & 9,177 & & $34 \cdot 36$ & 27.82 & 65.64 \\
\hline Bear Swamp & $3 \mathrm{~g}$ & II3 & 9.03 & 55.01 & & & & & & 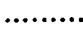 \\
\hline Westwood & $4 \mathrm{~b}$ & 114 & I 2.79 & $33 \cdot 3^{8}$ & & & & & & \\
\hline & $4 \mathrm{c}$ & 115 & 16.90 & $7 \cdot 3^{\circ}$ & 5,203 & 9,365 & 2. I I & 33.5 .1 & $26.2 \mathrm{I}$ & 66.49 \\
\hline Southtown & $58 \mathrm{~b}$ & 116 & 20.90 & 13.42 & 4,795 & 8,632 & $2.5^{2}$ & $3^{6.83}$ & $23.4 \mathrm{I}$ & 63.17 \\
\hline Kerrs Corners. & $58 \mathrm{e}$ & I 17 & 22. 13 & 8.01 & 5,120 & 9,215 & 2.35 & 32.62 & $24.6 \mathrm{I}$ & $67.3^{8}$ \\
\hline Kerrs Corners. & $58 \mathrm{k}$ & 118 & 21.89 & 7.72 & 4,982 & 8,968 & I. 59 & 36.70 & 28.98 & \\
\hline Danville. & $56 \mathrm{a}$ & I I9 & 28.14 & 8.62 & 4,953 & 8,915 & 2.45 & 33.14 & 24.52 & \\
\hline & $57 a$ & 120 & 21.63 & 15.82 & $4,3^{17}$ & $7,77^{2}$ & 2.27 & 36.35 & 20.53 & 63.65 \\
\hline Greenville & $59 \mathrm{~d}$ & I2I & 16.39 & 42.11 & & & $1.5^{8}$ & & & $\because$ \\
\hline Green & $59 \mathrm{~g}$ & I 22 & 6.09 & 42.80 & & & 1.88 & & & \\
\hline $\mathrm{Fe}$ & $60 a$ & I 23 & 14.72 & $40.8 \mathrm{I}$ & & & I. 56 & & & \\
\hline Mt. Herman & $60 \mathrm{e}$ & I 24 & 22.68 & I6.7 I & 4,568 & 8,224 & 2. I3 & 36.46 & I9.75 & 3.54 \\
\hline Buttsville & $61 \mathrm{e}$ & 125 & I6.82 & $10.4 \mathrm{I}$ & 4,863 & & 2.65 & $34.5^{I}$ & 24. JO & $5 \cdot 49$ \\
\hline Pigeon Swamp & $62 \mathrm{k}$ & 126 & 24.25 & 20.40 & 4,539 & 8,169 & 1.72 & 37.25 & 16.85 & 62.75 \\
\hline Pompton Plain. & $63 c$ & 127 & 13.82 & 38.50 & & & 1.48 & 55 & & 58.45 \\
\hline Pompton Plain. & $63 \mathrm{e}$ & 128 & I9. I7 & 13.64 & 77 & 8,579 & 2. I I & $4^{\mathrm{I}} \cdot 55$ & 27.91 & 8.45 \\
\hline 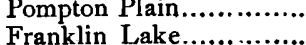 & $63 \mathrm{e}$ & $\begin{array}{l}\mathbf{1} 29 \\
-120\end{array}$ & II. 36 & $\begin{array}{r}52.19 \\
8.34\end{array}$ & & 8 & $\begin{array}{l}1.34 \\
2.03\end{array}$ & 36.28 & 27.9 & 63.72 \\
\hline $\begin{array}{l}\text { Franklin Lake ........... } \\
\text { Woodbridge }\end{array}$ & & & 12.50 & 0.34 & & & & & & \\
\hline & & & 18.82 & 10.40 & 4,926 & 8,865 & 2.62 & 34.40 & 24.00 & 65.60 \\
\hline Mo & & & 7.64 & $46.7 \mathrm{I}$ & & & 0.94 & & & \\
\hline
\end{tabular}

Present Uses in New Jersey.-The chief use of peat in New Jersey at present is in connection with the manufacture of fertilizer chiefly as a filler and for mixing with dried blood. The use of peat for this purpose presents certain advantages both from a mechanical and chemical standpoint, which render its use desirable. Where peat is used as a filler the fertilizer shows less tendency to become lumpy, so that a more even distribution on the soil is readily obtained. Since all peat contains varying amounts of nitrogen, its use as a filler increases the nitrogen content of the fertilizer. It is true, indeed, that many authorities deny any value to peat nitrogen as a source of food to plants, when directly applied to the soil. If their view is correct, the consumer has good grounds for objections, when the manufacturer includes the peat nitrogen in his guarantee, along 
with the high-grade organic nitrogen. Other authorities, however, assert that the peat nitrogen does become available for plant food, although not very rapidly, and that it has some money value for this purpose. However that may be, the fact remains that it is now used extensively for this purpose by manufacturers of fertilizer.

The Woodbridge Manufacturing Company have a plant located near Danville, on the Pequest Meadows, where the upper layer of peat is dug, dried and pulverized. The drying is done in an inclined cylindrical kiln, 30 feet long, 4 feet in diameter and takes from 15 to 20 minutes. The capacity of the plant was I I to I 2 tons per I2-hour day, but it has recently been enlarged.

The American Agricultural Chemical Company and the Alphano Company have recently started operations several miles northeast of Danville, on the west and east sides of the Pequest Meadows respectively.

Several attempts have been made to utilize peat as fuel. The most recent was made by the American Peat Coal Company, whose plant, located on the Bog and Vly Meadows, 2 miles west of Pequanac, was in operation during parts of 1904 and 1905. The freshly dug peat was put through a kneading and molding machine, of the type known as the Heinen Double Breaker, with a daily capacity of 50 tons. The peat after thorough mixing and kneading issued from the die in two bars, each $4 \frac{T}{2}$ inches square, which were cut by hand into briquettes, transferred on trays to the carrying belt which conveyed them to the drying house. No attempt was made to expell the water by pressure in the machine, but the briquettes were air-dried on shelves of wire netting for 2 to 4 days. The finished product was clean and hard, and could be handled without much breakage. The plant was never run to its full capacity and the market was mainly local, the fuel being used for domestic purposes chiefly. It sold at $\$ 3.00$ per ton. 